\title{
NASA/ADS
}

\section{Nonlinear filtering for sequential spacecraft attitude estimation with real data: cubature Kalman filter, unscented Kalman filter and extended Kalman filter.}

Show affiliations

\section{Veloso Garcia, Roberta; Pardal, Paula C. P. M.; Koiti Kuga, Helio}

The purpose of this work is to analyze the performance of the Cubature Kalman Filter, Unscented Kalman Filter and Extended Kalman Filter estimators in the attitude estimation problem when submitted to real attitude sensors data.The Extended Kalman Filter (EKF) is the most used nonlinear filtering algorithm for the attitude estimation in real time. The EKF is the nonlinear version of the Kalman Filter which linearizes about an estimate of the current mean and covariance. However, when the filter is subjected to poor conditions, the linearization of the system may not be efficient and lead to an estimation of low accuracy and divergence of the filter. The Unscented Kalman Filter (UKF) is an algorithm that was developed in order to avoid the linearizations required by the EKF. Basically, the UKF uses a set of points chosen deterministically, called "sigmapoints", to capture the probability distribution and generalizes to nonlinear system without the burdensome analytic derivation as in the EKF. More recently, the Cubature Kalman Filter (CKF) was proposed as an alternative estimation algorithm for general nonlinear systems. The CKF, which builds on the numerical-integration perspective of Gaussian filters, employs a third-degree spherical-radical cubature rule to compute Gaussian-weighted integrals, derivative-free nonlinear filtering algorithm with improved performance over the UKF in terms of estimation accuracy, numerical stability and computational costs.In this work, the application uses the real measurement data for orbit and attitude of the CBERS-2 (China Brazil Earth Resources Satellite) satellite. The attitude dynamical model is described by nonlinear equations involving the Euler angles. The attitude sensors available are two DSS (Digital Sun Sensors), two IRES (Infra-Red Earth Sensor), and one triad of mechanical gyros. The analyzes are based on the robustness of the filter, in relation to the precision, computational cost and convergence speed in attitude estimation. As the use of real data makes it impossible to compare the estimated results with the real attitude of the satellite, then the results obtained via EKF are taken as reference for comparison with the UKF and CKF. The results in this work 
show that, for the case studied in this article, the filters are very competitive and present advantages and disadvantages that should be evaluated according to the need of each problem.

\section{Publication:}

42nd COSPAR Scientific Assembly. Held 14-22 July 2018, in

Pasadena, California, USA, Abstract id. PSD.1-41-18.

\section{Pub Date:}

July 2018

\section{Bibcode:}

2018cosp...42E3523V

D Feedback/Corrections? (/feedback/correctabstract?bibcode=2018cosp...42E3523V) 